\title{
Correlation between baseline echographic features of endophthalmitis, microbiological isolates, and visual outcomes
}

This article was published in the following Dove Press journal:

Clinical Ophthalmology

19 April 2013

Number of times this article has been viewed

\author{
Aleksandra $\vee$ Rachitskaya \\ Harry W Flynn Jr \\ Yale L Fisher \\ Bernadete Ayres \\ Department of Ophthalmology, \\ Bascom Palmer Eye Institute, \\ University of Miami, Miller School \\ of Medicine, Miami, FL, USA
}

Background: The purpose of this study was to examine the baseline echographic features in culture-positive and culture-negative endophthalmitis and to correlate these echographic features with final visual outcomes.

Methods: We identified a retrospective noncomparative case series of patients with a clinical diagnosis of endophthalmitis and a baseline echographic examination between 1996 and 2010 at a single institution. Graded echographic features studied included: dense, moderate, and mild vitreous opacities; marked, moderate, and mild vitreous membranes; retinal detachment; and choroidal detachment. These were compared between culture-negative, coagulase-negative staphylococci, and other culture-positive cases of endophthalmitis, and were correlated with final visual outcomes.

Results: Of 149 patients reviewed, 60 were culture-negative, 26 grew coagulase-negative staphylococci, and 60 grew other culture-positive species. Three had multiple culture isolates. The presence of dense, moderate, and mild vitreous opacities, marked, moderate, and mild vitreous membranes, retinal detachment, and choroidal detachment was not significantly different between the three groups ( $P=0.86, P=0.56, P=0.34, P=0.45$, respectively). The presence of advanced echographic features of dense vitreous opacities, marked vitreous membranes, retinal detachment, and choroidal detachment was correlated with poorer visual acuity outcomes ( $P=0.005, P=0.0001, P<0.0001$, and $P<0.0001$, respectively).

Conclusion: No correlation could be made between echographic features and the organism. The presence of advanced echographic features, such as dense vitreous opacities, marked vitreous membranes, retinal detachment, and choroidal detachment, was correlated with worse visual outcomes.

Keywords: endophthalmitis, echography, retina, ultrasound

\section{Introduction}

Endophthalmitis results from an infectious organism gaining access to the eye via a variety of etiologies. In order to achieve the optimal treatment outcome, this sightthreatening condition requires prompt diagnosis and institution of the most effective treatment. Because the opacified media frequently prevents detailed visualization of the posterior segment, use of echography is often necessary. Echographic features in combination with clinical presentation aid in the diagnosis and influence clinical decision-making. Echographic features reported to be associated with endophthalmitis include vitreous opacities, vitreous membranes, retinal detachment, subhyaloid opacities, subretinal opacities, choroidal detachment, fundus thickening, and signs of orbital inflammation. ${ }^{1}$ The current study evaluated the echographic features of a
Correspondence: Harry W Flynn Jr Bascom Palmer Eye Institute, 900 NW 17th Street, Miami, FL 33I36, USA

Tel + I 3053266118

Fax +I 3053266417

Email hflynn@med.miami.edu 
series of patients with endophthalmitis at a university referral center.

\section{Materials and methods}

The current study was an institutional review board-approved retrospective, noncomparative case series identified from 1996 to 2010 at Bascom Palmer Eye Institute (BPEI). The echography department database was reviewed to identify patients with clinically suspected endophthalmitis who underwent an echography examination. Only patients who had an intraocular culture specimen obtained by anterior chamber aqueous paracentesis, vitreous tap at the bedside, or during vitrectomy were included. The medical records as well as the archived microbiological culture results for these patients were reviewed.

Standardized echography was performed on all study patients by staff of the BPEI echography department. V2 and V3 units (Ellex Medical Lasers Ltd, Adelaide, Australia) were used for standardized diagnostic A-scan and contact B-scan echography. Echography performed on the day of presentation or the first echography performed after initial presentation was considered to be the baseline examination. Baseline echographic features documented included: absent, mild, moderate, and dense vitreous opacities; absent, mild, moderate, and marked vitreous membranes; posterior vitreous detachment; retinal detachment; subretinal opacities; choroidal detachment; degree of fundus thickening; and any additional echographic features noted by the echographer. The echographic examinations and interpretations were performed by certified echography technicians and confirmed by a physician.

The patient data collected included: baseline demographic features, including patient gender and age; presenting visual acuity, intraocular pressure, presence of pain and hypopyon, and etiology of endophthalmitis; echographic findings as characterized above; culture isolates; initial and subsequent management; and time between treatment and echographic examination. In addition to initial visual acuity, visual acuity at months $1,3,6$, and 12 , along with the last known visual acuity were recorded. Final visual acuity was defined as visual acuity at 12 months or last recorded, whichever came earlier.

Intraocular samples for microbiological diagnosis were obtained by standard anterior chamber aqueous paracentesis, vitreous tap at the bedside, or during vitrectomy. During vitrectomy, either the undiluted vitreous sample or vitreous wash from the vitrectomy cassette were submitted. The samples were cultured in a standard fashion using blood agar, chocolate agar, Sabouraud agar, Lowenstein-Jensen agar, or thioglycolate broth. Each positive culture was recorded separately. For analysis, the cases were divided in three groups, ie, culture negative, coagulase-negative staphylococci, and other culture-positive cases. Three patients with multiple culture isolates were excluded when analyzing the relationship between echographic features and microbiological outcomes.

A standard $\log$ MAR scale was used for the statistical analysis. Patients with count fingers visual acuity were assigned a $\log$ MAR value of 2.3, hand motion 2.6, light perception 3.0, and no light perception 4.0. ${ }^{2}$ Eyes that were enucleated or eviscerated were considered to be no light perception. $P<0.05$ was deemed to be statistically significant.

\section{Results}

Review of the echography department database identified 149 patients with clinically suspected endophthalmitis who underwent echography. Of these, 89 were intraocular (vitreous or anterior chamber) culture-positive and 60 were culture-negative.

\section{Baseline clinical features}

Of the 149 patients, 78 were female and 71 were male. The mean age at presentation was 66 years. Mean visual acuity was $2.7 \log$ MAR (hand motion to light perception) and median visual acuity was 2.6 logMAR (hand motion). Mean intraocular pressure was $19 \mathrm{mmHg}$. Pain was reported by 103 (69\%) patients and hypopyon was documented in $104(70 \%)$. Endophthalmitis occurred after cataract surgery in 50 patients (33.6\%), after glaucoma filtration surgery in $23(15.4 \%)$, after penetrating keratoplasty surgery in $18(12.1 \%)$, after trauma in $17(11 \%)$, and after intravitreal injection in seven $(4.7 \%)$. Endophthalmitis of endogenous origin was documented in 19 cases $(12.8 \%)$. In addition, 12 cases were associated with corneal ulcer, one with orbital cellulitis, and two were of unknown cause. As initial management, tap and inject was performed in 111 patients $(74.5 \%)$, primary pars plana vitrectomy in 21 (14\%), and primary evisceration or enucleation in $16(11 \%)$ (Table 1). The initial treatment in one patient is unknown. Mean final visual acuity was 2.6 logMAR (count fingers) and the median was $2.7 \log$ MAR (hand motion).

\section{Echography in culture-negative, coagulase-negative staphylococci, and other culture-positive cases}

The echographic features studied included vitreous opacities, vitreous membranes, retinal detachment, and choroidal 


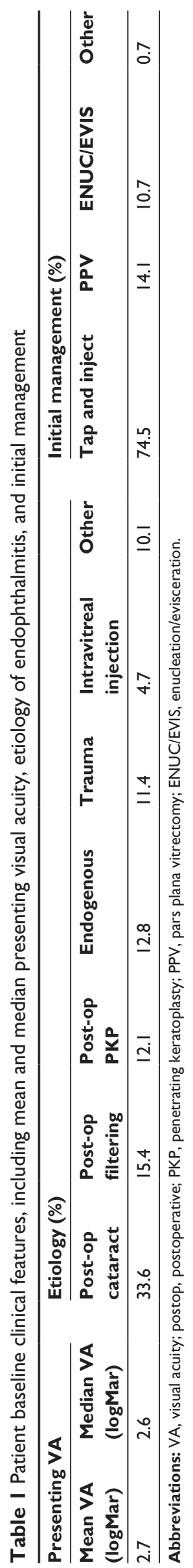

detachment (Figure 1). These features were correlated with three groups of microbiological isolates, ie, culture-negative $(n=60)$, coagulase-negative staphylococci $(n=26)$, and other culture-positive species $(n=60)$.

In culture-negative samples $(n=60), 26$ patients $(43.3 \%)$ had dense vitreous opacities and $25(41.7 \%)$ had marked vitreous membranes. Posterior vitreous detachment was present in 13 patients $(21.7 \%)$, retinal detachment in $21(35 \%)$, and choroidal detachment in 11 (18.3\%) (Figure 2 and Table 2). Mean and median final visual acuities were 2.1 $\log$ MAR $(2 / 200$ to $1 / 200)$ and $2.75 \operatorname{logMAR}$ (hand motion to light perception), respectively (Table 3 ).

Eighty-nine patients had culture-positive endophthalmitis. In three cases, two organisms grew from the same sample, and these were excluded from the analysis. The remaining 86 cases were analyzed by microbial isolate and echographic findings. These cases included coagulase-negative staphylococci $(n=26)$, fungal species $(n=10)$, streptococcal species $(\mathrm{n}=8)$, methicillin-sensitive Staphylococcus aureus $(\mathrm{n}=6)$, Pseudomonas aeruginosa $(\mathrm{n}=6)$, Enterococcus faecalis $(\mathrm{n}=5)$, Bacillus cereus $(\mathrm{n}=5)$, methicillin-resistant Staphylococcus aureus $(\mathrm{n}=3)$, Serratia marcescens $(\mathrm{n}=3)$, Klebsiella pneumoniae $(\mathrm{n}=2)$, Enterobacter cloacae $(\mathrm{n}=2)$, Propionibacterium acnes $(\mathrm{n}=2)$, Stenotrophomonas maltophilia $(\mathrm{n}=1)$, coagulase-positive Staphylococcus intermedius $(\mathrm{n}=1)$, Proteus mirabilis $(\mathrm{n}=1)$, Pantoea agglomerans $(\mathrm{n}=1)$, Mycobacterium chelonae $(\mathrm{n}=1)$, Morganella morganii $(\mathrm{n}=1)$, Haemophilus influenzae $(\mathrm{n}=1)$, and Corynebacterium aquaticum $(\mathrm{n}=1)$.

The presence of echographic features was documented for each organism studied, but given the small number of specific isolates, the culture-positive organisms were divided
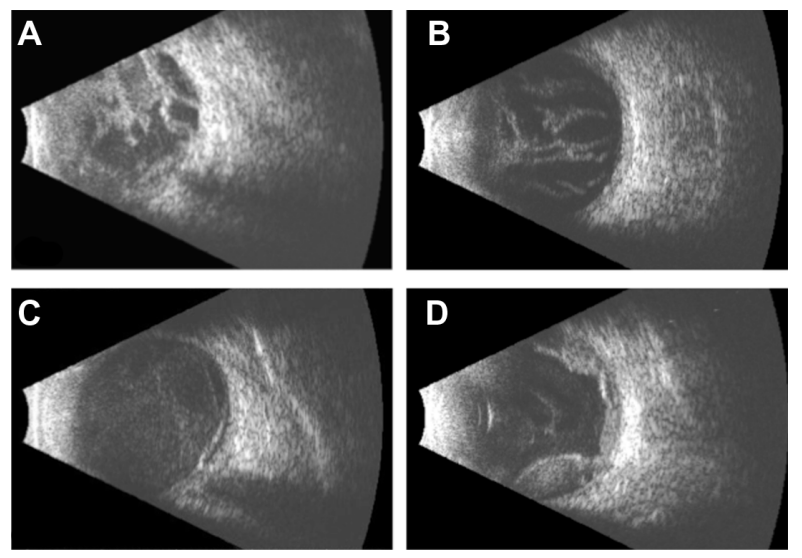

Figure I Representative echographic features of $(\mathbf{A})$ dense vitreous opacities with retinal and choroidal detachment, (B) marked vitreous membranes, (C) retinal detachment with retinal opacities, and (D) choroidal detachment (with retinal opacities). 
Dense vitreous opacities

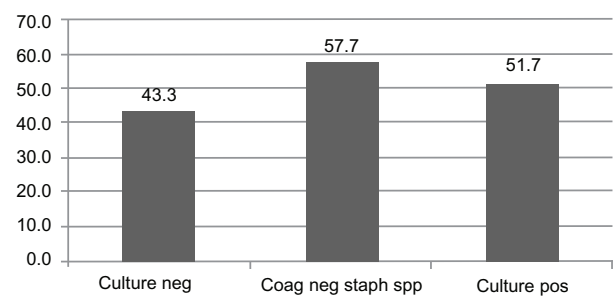

Retinal detachment

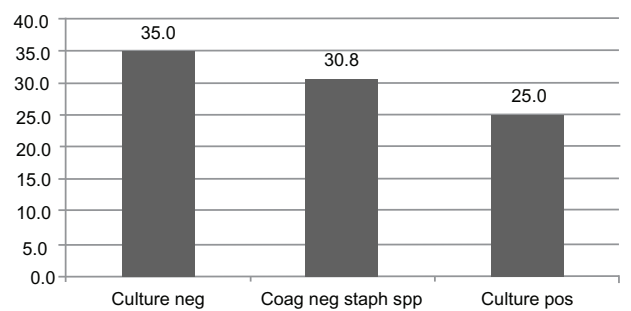

Marked vitreous membranes

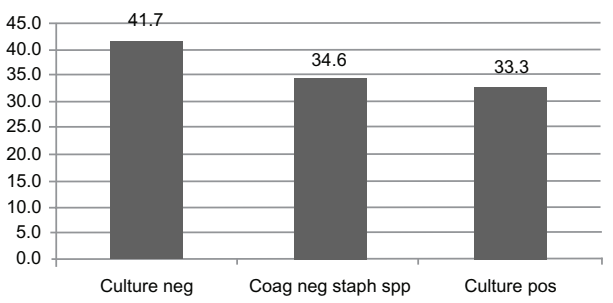

Choroidal detachment

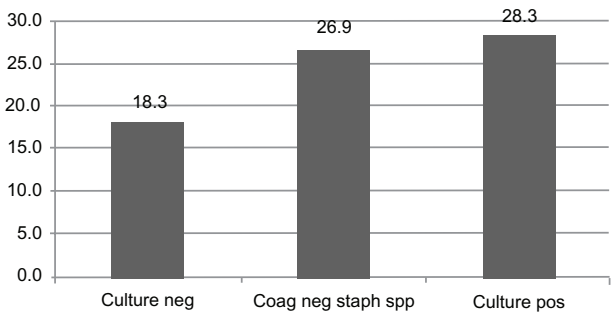

Figure 2 Advanced echographic features among culture-negative, coagulase-negative staphylococci, and other culture-positive endophthalmitis. Percentage of dense vitreous opacities (A), marked vitreous membranes (B), retinal detachment (C), and choroidal detachment (D) in culture-negative endophthalmitis cases, in coagulase-negative staphylococci, and other culture-positive endophthalmitis cases.

Abbreviation: coag neg staph spp, coagulase-negative staphylococci.

into two groups, ie, coagulase-negative staphylococci and other culture-positive organisms.

Of 26 coagulase-negative staphylococci cases, 15 (57.7\%) had dense vitreous opacities. Marked membranes were present in nine $(34.6 \%)$. Posterior vitreous detachment was documented in seven (26.9\%) cases. Retinal detachment was seen in eight $(30.8 \%)$ and choroidal detachment in seven (26.9\%) (Figure 2 and Table 2). The mean and median final visual acuities were 1.4 $\log$ MAR (8/200) and $1.3 \log$ MAR (20/400), respectively (Table 3).

In other culture-positive cases $(\mathrm{n}=60), 31(51.7 \%)$ had dense vitreous opacities. Marked vitreous membranes were present in 20 (33.3\%). Posterior vitreous detachment was present in $12(20 \%)$. Retinal detachment was documented in $15(25 \%)$ and ciliochoroidal detachment in $17(28.3 \%)$ patients (Figure 2 and Table 2). Mean and median visual acuity was $2.1 \log$ MAR $(2 / 200$ to $1 / 200)$ and 2.7 logMAR (hand motion), respectively (Table 3 ).

When comparing the presence of baseline echographic findings of dense, moderate, mild, and absent vitreous opacities among culture-negative cases, coagulase-negative staphylococci, and other culture-positive species, there were no significant differences found between the three groups of microbiological isolates ( $P=0.86$, Kruskal-Wallis test). Similarly, no differences were found between the three groups when comparing the presence of marked, moderate, mild, and absent vitreous membranes $(P=0.56$, Kruskal-Wallis test). The rates of retinal detachment in the three groups showed no statistically significant differences $(P=0.34$, Chi-square, partition test). Further, there was no statistically significant difference between the three groups for presence of choroidal detachment ( $P=0.45$, Chi-square, partition test). Coagulasenegative staphylococcai species had the best visual acuity outcome of the three groups of microbiological isolates $(P=0.013$, analysis of variance $)$.

\section{Correlation between echographic features and visual acuity outcome}

The final visual acuity outcome was known in 148 patients. This was poor overall, with a mean visual acuity of $1.9 \log$ MAR (3/200 to 2/200) and a median of $2.7 \log$ MAR (hand motions). The presence of characteristic echographic features was correlated with the final visual outcome.

In patients with dense vitreous opacities $(n=74)$, mean final visual acuity was $2.2 \log$ MAR (2/200 to $1 / 200)$, which was significantly worse than in patients with moderate, or mild opacities $(\mathrm{n}=74)$ who had a final mean visual acuity of $1.75 \log$ MAR (4/200 to $3 / 200, P=0.005$, $t$-test, Figure 3 ). No patients with absent vitreous opacities were identified. Overall, more advanced opacities were associated with worse final visual acuity ( $P=0.0052$, Spearman's correlation coefficient).

Similarly, final visual acuity in the 56 patients with marked vitreous membranes was significantly worse than in the 92 patients with moderate, mild, or absent vitreous membranes (2.4 $\log$ MAR [ $1 / 200$ to count fingers] and $1.75 \log$ MAR [4/200 to $3 / 200]$, respectively, 


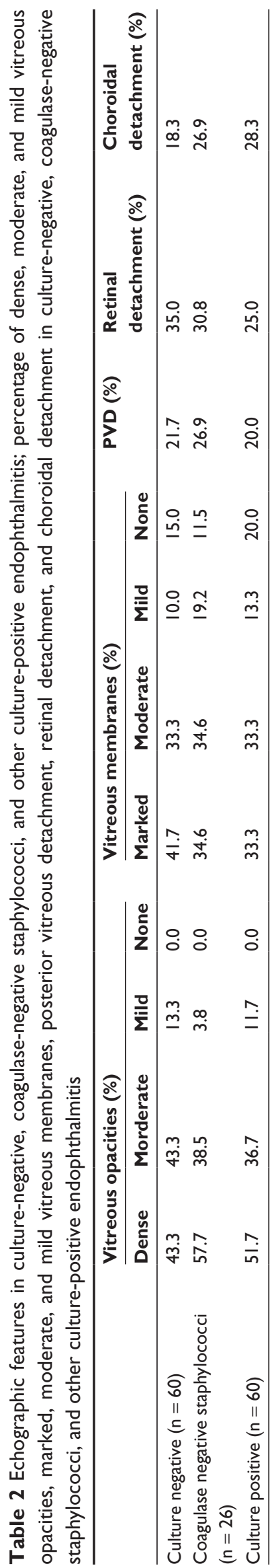

Table 3 Visual acuity in among culture-negative, coagulase-negative staphylococci species, and other culture-positive endophthalmitis; final mean and median visual acuities in culture-negative endophthalmitis cases, in coagulase-negative staphylococci, and other culture-positive endophthalmitis cases

\begin{tabular}{lll}
\hline & $\begin{array}{l}\text { Mean final } \\
\text { visual acuity }\end{array}$ & $\begin{array}{l}\text { Median final } \\
\text { visual acuity }\end{array}$ \\
\hline $\begin{array}{l}\text { Culture negative } \\
\text { Coagulase negative }\end{array}$ & $2 / 200$ to $\mathrm{I} / 200$ & $\mathrm{HM}$ to $\mathrm{LP}$ \\
$\begin{array}{l}\text { staphylococci } \\
\text { Culture positive }\end{array}$ & $8 / 200$ & $20 / 400$ \\
\hline
\end{tabular}

Abbreviations: HM, hand motion; LP, light perception.

$P=0.0001, t$-test). As with the vitreous opacities, the more advanced the degree of vitreous membranes, the poorer the final visual acuity was noted $(P<0.0001$, Spearman's correlation coefficient, Figure 3).

When cases with retinal detachment $(n=43)$ were compared with those without $(\mathrm{n}=105)$, there was a statistically significant difference in final mean visual acuity (2.5 $\log$ MAR [1/200 to count fingers] and $1.8 \log$ MAR [3/200], respectively, $P<0.0001, t$-test, Figure 3).

The presence of choroidal detachment $(\mathrm{n}=34)$ resulted in a mean final visual acuity of $2.4 \log$ MAR (1/200 to count fingers) that was significantly worse when compared with the 114 cases in which no choroidal detachment was documented (mean final visual acuity $1.9[3 / 200$ to $2 / 200], P=0.0038$, $t$-test, Figure 3).

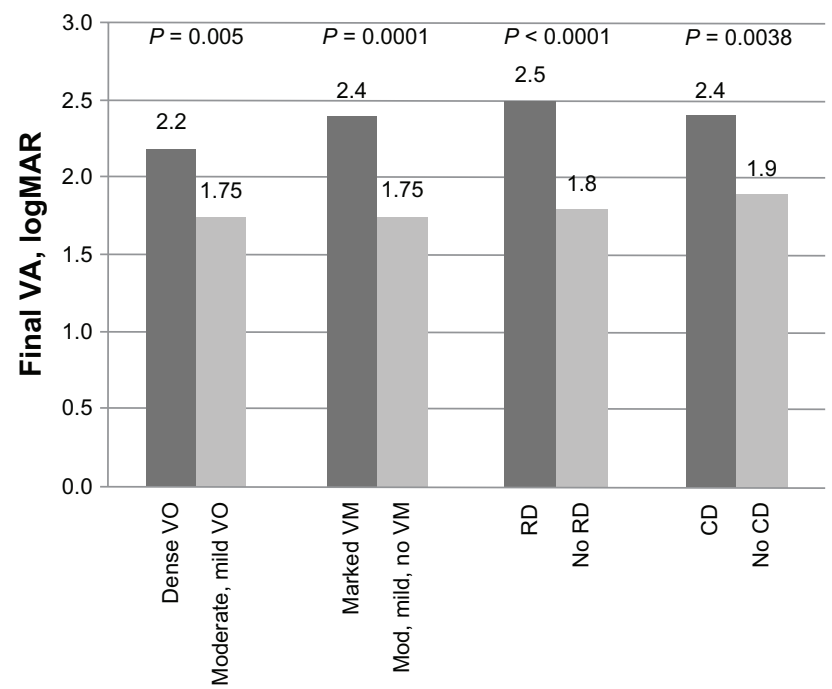

Figure 3 Advanced echographic features and visual acuity outcomes.

Notes: Statistically significant difference in final visual acuity (logMAR) in cases with dense as compared with moderate or mild vitreous opacities, marked as compared with moderate, mild, or no vitreous membranes, with and without retinal detachment, and with and without choroidal detachment.

Abbreviations: $\mathrm{VA}$, visual acuity; $\mathrm{VO}$, vitreous opacities; VM, vitreous membranes; $\mathrm{RD}$, retinal detachment; $C D$, choroidal detachment. 


\section{Discussion}

The echographic features of endophthalmitis have been described previously. ${ }^{1,3-6}$ Ophthalmic echography is a valuable diagnostic adjunct to opaque media in patients with clinically suspected endophthalmitis. In the Endophthalmitis Vitrectomy Study, echography was performed to determine if choroidal detachment, retinal detachment, or both, were present when the ocular media precluded direct visualization of the posterior pole. ${ }^{7}$ However, the question remains as to whether the findings seen on initial echographic examination can aid the physician in more than a binary fashion in terms of increasing or decreasing suspicion for infectious endophthalmitis.

Previous studies correlating the initial echographic findings in infectious endophthalmitis with visual prognosis and causative microorganisms have not been conclusive. In one study that examined 137 eyes with 102 positive cultures, the authors noted that an echographically clear vitreous may be suggestive of early streptococcal or culture-negative endophthalmitis. Severe vitreous opacities, vitreous membranes, and complete posterior vitreous detachment appeared more likely to be found in streptococcal endophthalmitis. Choroidal detachment occurred more commonly in Gram-negative endophthalmitis. The authors concluded that ocular echography is of limited usefulness in the diagnosis of infectious endophthalmitis because none of the findings were specific. ${ }^{8}$ Another study examined 28 eyes and showed that the presence of dense vitreous opacities in endophthalmitis associated with cataract surgery was correlated significantly with isolation of Staphylococcus aureus and Pseudomonas aeruginosa. ${ }^{9}$

Coagulase-negative staphylococci are the most frequent microbial isolates in culture-positive endophthalmitis. ${ }^{10} \mathrm{In}$ the current study, coagulase-negative staphylococci had the best visual outcome as compared with culture-negative and other culture-positive endophthalmitis. However, when the presence of dense vitreous opacities, marked vitreous membranes, retinal detachment, and choroidal detachment was compared between the three groups, no difference was found. On the other hand, the presence of dense vitreous opacities, marked vitreous membranes, retinal detachment, and choroidal detachment correlated with worse final visual acuity. This indicates that, although the presence of these advanced echographic findings correlates with worse visual prognosis, no correlation can be made with the possible causative organism.

A limitation of this study is its relatively small sample size. Further, not all patients with suspected endophthalmitis undergo echography. Clinically complicated cases are more likely to undergo echographic examination. Therefore, the sample studied might not have encompassed all patients with endophthalmitis, in particular missing those with milder presentation, less virulent organisms, and less advanced echographic findings. Moreover, the examinations are performed by different echography department technicians. Echographic pattern recognition is frequently difficult because of the rapidly changing anatomic patterns that may accompany endophthalmitis. ${ }^{4,5}$ In the current study, of 59 culture-negative patients for whom data were available, the mean interval between treatment and initial ultrasound was 0.8 days (median 0 days). In 85 culture-positive patients for whom these data were available, the mean interval between treatment and initial ultrasound was 1.4 days (median 0 days). Partial or complete vitreous face separations as well as neurosensory retina and choroidal separation patterns are often encountered, and difficult to recognize within significant reflective tissue. Further, it is often difficult to interpret echographic findings, because of the wide range of acoustic impedance mismatch within vitreous cavity tissues, dependent upon inflammatory reaction. Patterns seen during echographic examination range from barely visible, mildly reflective spot and dot-like images, to extensive, elaborate, and strongly reflective vitreous conglomerations appearing as veil-like sheets or curtains, which are almost "membranous" in form. ${ }^{4-6,11}$ In the current study, we graded the appearance of vitreous opacities (dense, moderate, mild, none) and membranes (marked, moderate, mild, none). It is important to remember to compare the affected eye with the uninvolved eye using real-time kinetic evaluation and observe "normal" vitreous movement. ${ }^{12}$

Echography patterns coupled with clinical history and ophthalmic examination lend credence to an echography pattern diagnosis consistent with endophthalmitis. It is important to bear in mind that intraocular reflections are those of acoustic impedance tissue mismatch and not cellular pathology. Echography patterns alone are not pathognomonic of a causative organism, but advanced echographic features are associated with poorer visual outcome.

\section{Acknowledgments}

This research is funded in part by a center core grant from the National Institutes of Health (P30EY014801), an unrestricted grant from Research to Prevent Blindness, and a grant from the Department of Defense (W81XWH-09-1-0675). The authors thank Manuel Ayres, Wei Shi, and Joyce Schiffman for their assistance with the statistical analysis. 


\section{Disclosure}

The authors report no conflicts of interest in this work, and have no proprietary interest in any materials mentioned herein.

\section{References}

1. Byrne SF, Green RL. Ultrasound of the Eye and Orbit, 2nd ed. St Louis, MO: Mosby Year Book; 2002.

2. Grover S, Fisherman GA, Anderson RJ, et al. Visual acuity impairment in patients with retinitis pigmentosa at age 45 years or older. Ophthalmology. 1999;106:1780-1785.

3. Oksala A. The echogram in vitreous opacities. Am J Ophthalmol. 1960; 49:1301-1307.

4. Marchini G, Pagliarusco A, Tosi R, Castagna G. Ultrasonographic findings in endophthalmitis. Acta Ophthalmol Scand. 1995;73:446-449.

5. Kohanim S, Daniels AB, Huynh N, Eliott D, Chodosh J. Utility of ocular ultrasonography in diagnosing infectious endophthalmitis in patients with media opacities. Semin Ophthalmol. 2012;27:247-250.
6. Maneschg O, Csakany B, Nemeth J. Ultrasonographic findings in endophthalmitis following cataract surgery: a review of 81 cases. Ophthalmologe. 2009;106:1012-1015. German.

7. Wisniewski SR, Capone A, Kelsey SF, Groer-Fitzgerald S, Lambert HM, Doft BH. Characteristics after cataract extraction or secondary lens implantation among patients screened for the Endophthalmitis Vitrectomy Study. Ophthalmology. 2000;107:1274-1282.

8. Dacey MP, Valencia M, Lee MB, et al. Echographic findings in infectious endophthalmitis. Arch Ophthalmol. 1994;112:1325-1333.

9. Patil RTD, Tewari HK, Sharma YR, Nainiwal S, Verma LK, Satpathy G. Relationship of echographic findings with visual outcomes in postcataract surgery endophthalmitis. Ann Ophthalmol. 2004;36:12-16.

10. Lalwani GA, Flynn HW Jr, Scott IU, et al. Acute-onset endophthalmitis after clear corneal cataract surgery (1996-2005). Clinical features, causative organisms, and visual acuity outcomes. Ophthalmology. 2008;115:473-476.

11. Chan IM, Jalkh AE, Trempe CL, Tolentino FI. Ultrasonographic findings in endophthalmitis. Ann Ophthalmol. 1984;16:778-784.

12. Fisher YL, Slakter JS, Friedman RA, Yannuzzi LA. Kinetic ultrasound evaluation of the posterior vitreoretinal interface. Ophthalmology. 1991;98:1135-1138.
Clinical Ophthalmology

\section{Publish your work in this journal}

Clinical Ophthalmology is an international, peer-reviewed journal covering all subspecialties within ophthalmology. Key topics include: Optometry; Visual science; Pharmacology and drug therapy in eye diseases; Basic Sciences; Primary and Secondary eye care; Patient Safety and Quality of Care Improvements. This journal is indexed on

Submit your manuscript here: http://www.dovepress.com/clinical-ophthalmology-journal

\section{Dovepress}

PubMed Central and CAS, and is the official journal of The Society of Clinical Ophthalmology (SCO). The manuscript management system is completely online and includes a very quick and fair peer-review system, which is all easy to use. Visit http://www.dovepress.com/ testimonials.php to read real quotes from published authors. 